\title{
Pitfall in the high-throughput quantification of whole blood cyclosporin A using liquid chromatography-tandem mass spectrometry
}

\author{
Michael Vogeser ${ }^{1, *}$ and Ute Spöhrer ${ }^{2}$ \\ ${ }^{1}$ Institute of Clinical Chemistry, \\ ${ }^{2}$ Pharmacy Department, \\ Hospital of the University of Munich, Munich, \\ Germany
}

\begin{abstract}
In a growing number of laboratories the technique of liquid chromatography-tandem mass spectrometry is used for the quantification of cyclosporin $A$ in whole blood, employing cyclosporin $D$ as the internal standard. Cyclosporin A is extensively metabolized in vivo; in liquid chromatography-tandem mass spectrometry respective metabolites can give rise to both parent and product ions that are isobaric with ions commonly used for the detection of cyclosporin $A$ and cyclosporin D, respectively. In this article it is demonstrated that limited chromatography with co-elution of such metabolites together with cyclosporin A and cyclosporin D can lead to incorrect results.
\end{abstract}

Keywords: cyclosporin A; cyclosporin D; liquid chromatography-tandem mass spectrometry; specificity.

In tandem-mass spectrometry the detection of target analytes is based on their physical decomposition behavior; the molecular mass-specific selection of an intact precursor ion of an analyte and of the respective product ion results in very high analytical specificity. This high degree of specificity can limit the requirements of sample preparation and chromatography and may enable "high-throughput" routine methods. However, the specificity of tandem-mass spectrometry should not be assumed to be absolute and unlimited. Given the complexity of human body fluids as a matrix and the extensive metabolization of certain target analytes, the specificity of liquid chromatography-tandem mass spectrometry (LC-MS/MS) may be a critical issue. This is demonstrated by an observation reported here.

With the intention of switching the quantification of whole blood cyclosporin A (CsA) from immunoassay technology to LC-MS/MS, we decided to modify the LC-MS/MS method that we currently use for the quan-

*Corresponding author: Michael Vogeser, Institute of Clinical Chemistry, Hospital of the University of Munich, 81366 Munich, Germany

Phone: +49-89-7095-3221, Fax: +49-89-7095-3240,

E-mail: michael.vogeser@med.uni-muenchen.de tification of sirolimus (1) by additional acquisition of multiple reaction monitoring (MRM) mass transitions of CsA and cyclosporin D (CsD, internal standard), respectively (both compounds were donated by Novartis, Grenzach-Whylen, Germany). As a modification of our originally published method (1), extended chromatography was implemented, resulting in a retention time of approximately $3.4 \mathrm{~min}$ for sirolimus [isocratic elution after on-line solid phase extraction; Lichrospher $100-\mathrm{RP}-18120 \times 4 \mathrm{~mm}, 5 \mu \mathrm{m}$ column (Maisch, Ammerbuch, Germany) kept at $35^{\circ} \mathrm{C}$; mobile phase $90 \%$ methanol, $10 \% \quad 0.5 \mathrm{mM}$ ammonium acetate; flow $0.85 \mathrm{~mL} / \mathrm{min}$, post-column split 1:10]. Based on product ion scans of $\mathrm{Cs} A$ and $\mathrm{CsD}$, respectively, and in accordance with previously reported methods (2-5) the following MRM mass transitions were selected for CsA quantification: CsA, $1220 \rightarrow 1203$; and CsD (internal standard), $1234 \rightarrow 1217$. The respective parent ions represent the ammonium adducts of the compounds; the product ions arise from loss of the ammonium ion. In method validation, an inter-assay coefficient of variation of $8.4 \%$ was found for a CsA concentration of $67 \mu \mathrm{g} / \mathrm{L}$ and $5.1 \%$ for $332 \mu \mathrm{g} / \mathrm{L}$ $(n=21)$; the signal/noise ratio in a calibration sample with a concentration of $15.6 \mu \mathrm{g} / \mathrm{L}$ was approximately 70. The method was linear over a calibration range from 15.6 to $500 \mu \mathrm{g} / \mathrm{L}$ and no ion suppression by matrix effects was found.

When calibration materials (obtained by spiking hemolyzed drug-free whole blood with CsA) were analyzed under these conditions, one single peak was found for CsA and CsD in the respective MRM traces (Figure 1A; retention time approx. $4.3 \mathrm{~min}$ for CsA, and $4.7 \mathrm{~min}$ for $\mathrm{CsD}$ ). In contrast, when whole blood samples from CsA-treated patients were analyzed, we noted an additional peak with a distinctly shorter retention time of approximately $3.4 \mathrm{~min}$ in the MRM traces of both CsA and CsD (Figure 1B). These early eluting peaks probably correspond to more hydrophilic endogenously formed metabolites of CsA sharing the MRM transitions selected for the detection of CsA and CsD. Indeed, several metabolites of CsA have been reported that might be ionized to molecules that are isobaric with $\mathrm{CsA}$ and $\mathrm{CsD}$, respectively $(6,7)$.

Early eluting peaks were observed in all samples from CsA-treated patients, but not in any sample from patients receiving $\mathrm{CsA}$-free immunosuppression ( $\mathrm{n}>100$ each).

A quantification series of 10 samples from CsAtreated patients was performed based on the integration of peaks with the typical retention times of 


\section{A}



C

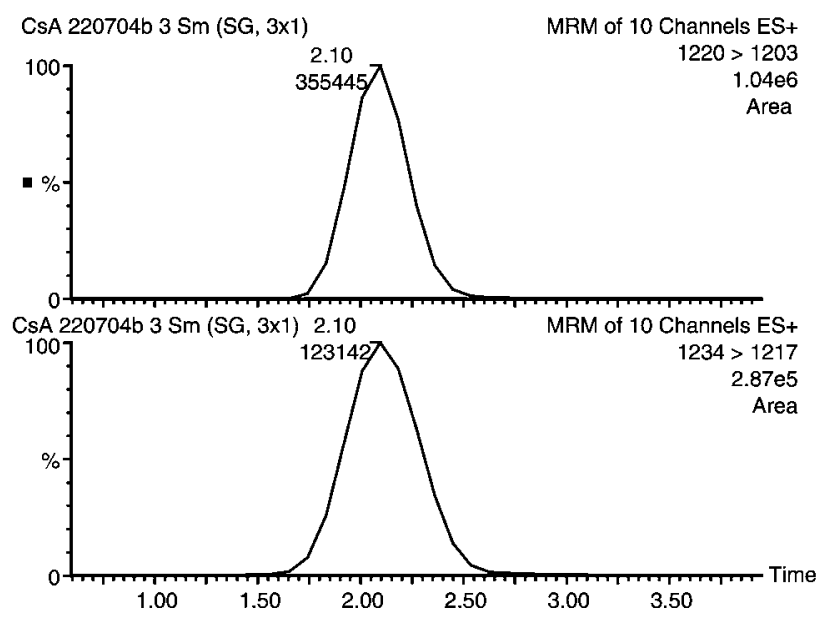

B

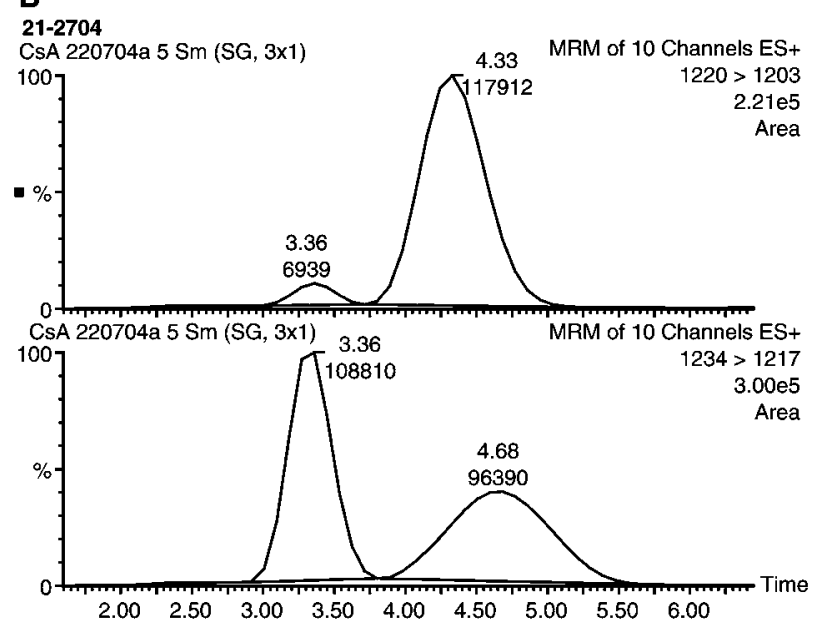

D

21-2704

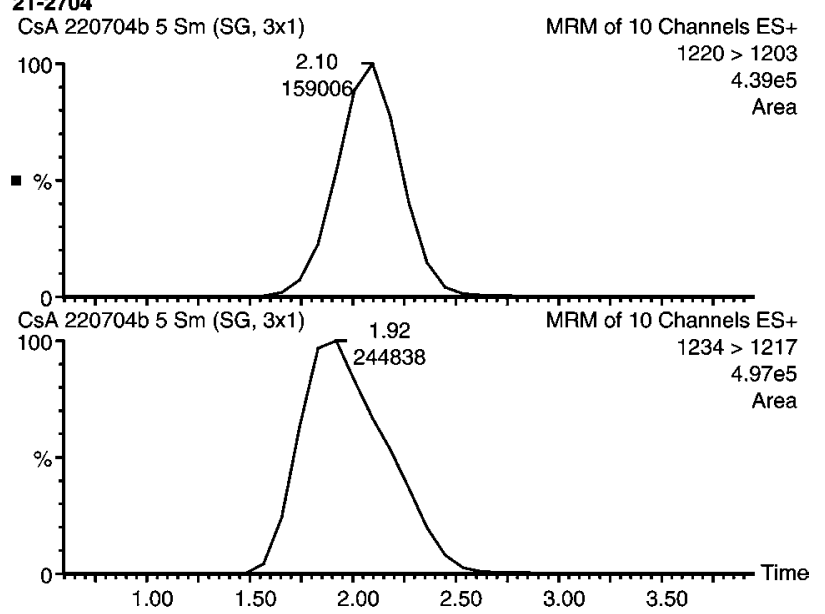

Figure 1 Multiple reaction monitoring chromatograms of two samples analyzed for cyclosporin A by LC-MS/MS. Spiked calibration sample (250 $\mu \mathrm{g} / \mathrm{L} ; A)$ and patient's sample (B) applying extended chromatography (C18-RP $125 \times 4 \mathrm{~mm}$ column); identical spiked calibration sample (C) and patient's sample (D) applying limited chromatography (C18-RP $75 \times 3 \mathrm{~mm}$ column). Cyclosporin A, mass transition $1220 \rightarrow 1203$; cyclosporin D (internal standard), mass transition $1234 \rightarrow 1217$. Y-Axis represents relative intensity of the signal with respect to the base peak of the chromatogram. Cyclosporin $A$ concentration of the calibration sample, $250 \mu \mathrm{g} / \mathrm{L}$; concentration of the patient's sample, $107 \mu \mathrm{g} / \mathrm{L}$.

CsA and CsD (4.3 and $4.7 \mathrm{~min}$ ) that were baselineseparated from the respective early eluting peaks; six-point calibration with spiked whole blood as the calibration material was performed (15.6-500 $\mu \mathrm{g} / \mathrm{L})$. Close agreement between LC-MS/MS results and the results of an immunoassay (SYVA, Dade Behring, Marburg, Germany) was found ( $r=0.99$; mean concentrations found: LC-MS/MS, $134 \mu \mathrm{g} / \mathrm{L}$; immunoassay, $137 \mu \mathrm{g} / \mathrm{L})$. As an experiment, the same series was re-analyzed using a shorter analytical column (identical column material, $75 \times 3 \mathrm{~mm}$ ) but otherwise identical chromatographic and mass spectrometric conditions, resulting in a substantially reduced analytical run time. Under these simplified chromatographic conditions, CsA and CsD co-eluted with the unspecified, more hydrophilic metabolites of CsA; one single peak was found and integrated in both MRM traces (Figure 1C, D), both in calibration samples and patient samples. The retention time of this single peak was approximately $2.1 \mathrm{~min}$, which is in agreement with previously described methods for the quantification of CsA employing $\mathrm{CsD}$ as the internal standard and the same mass transitions as applied here (2-5). The quantitative results obtained in this series using limited chromatography differed markedly from those obtained with extended chromatography and from the immunoassay results (Table 1 ). The mean concentration found by the LC-MS/MS method using limited chromatography was $58 \mu \mathrm{g} / \mathrm{L}$ [Pearson's $r=0.76$ between CsA results obtained with extended vs. limited chromatography; linear regression, (limited chromatography) $=0.16 \times($ extended chromatography) $+36.7 \mu \mathrm{g} / \mathrm{L}]$. In our experiment, omission of proper chromatographic separation resulted in a short run-time but obviously incorrect CsA results, although identical mass spectrometric conditions were applied.

Since ammonium adducts of both CsA and CsD give rise to several different product ions, we tried to find MRM transitions that might be specific for the respective compounds, but for all transitions investigated, non-compound specific peaks with different 
Table 1 Results of cyclosporin A quantification of 10 patients' whole blood samples obtained by LC-MS/MS applying cyclosporin $\mathrm{D}$ as the internal standard with different HPLC columns and by immunoassay (Dade Behring, SYVA).

\begin{tabular}{|c|c|c|c|}
\hline \multirow{2}{*}{$\begin{array}{l}\text { Sample } \\
\text { no. }\end{array}$} & \multicolumn{2}{|c|}{ Chromatography, $\mu \mathrm{g} / \mathrm{L}$} & \multirow{2}{*}{$\begin{array}{l}\text { Immunoassay, } \\
\mu \mathrm{g} / \mathrm{L}\end{array}$} \\
\hline & Extended & Limited & \\
\hline 1 & 107 & 63 & 101 \\
\hline 2 & 376 & 91 & 433 \\
\hline 3 & 36 & 37 & 34 \\
\hline 4 & 87 & 56 & 80 \\
\hline 5 & 171 & 100 & 150 \\
\hline 6 & 74 & 41 & 66 \\
\hline 7 & 254 & 65 & 277 \\
\hline 8 & 76 & 43 & 64 \\
\hline 9 & 45 & 35 & 43 \\
\hline 10 & 115 & 49 & 122 \\
\hline
\end{tabular}

Extended chromatography, C18-RP $125 \times 4 \mathrm{~mm}$; limited chromatography, C18-RP $75 \times 3 \mathrm{~mm}$.

retention times were found in patient samples if extended chromatography was applied. Our findings were confirmed with two different LC-MS/MS systems from the same manufacturer (Quattro LC and Quattro Ultima Pt, Waters Micromass, Manchester, UK) but were not tested with instruments from other manufacturers.

Based on the protonated molecular ion of CsA (formed with lower yield compared to the ammonium adduct), a MRM transition of CsA was found that was compound-specific in extended chromatography of patient samples $(1203 \rightarrow 425$; collision energy, $60 \mathrm{~V})$; this transition has been described previously (8). In contrast, no specific MRM transition was found for protonated CsD.

Using the specific CsA transition $1203 \rightarrow 425$ and 32desmethoxyrapamycin (Sigma-Aldrich, Deisenhofen, Germany; catalogue no. D6691; transition 901 $\rightarrow$ 834) as a common internal standard for both sirolimus and CsA measurement, we found it possible to quantify CsA under the simplified chromatographic conditions described $(75 \times 3 \mathrm{~mm}$ column). This assay had a reduced analytical run time of $3.5 \mathrm{~min}$. However, coefficients of variation clearly above $10 \%$ were found for validation quality control materials. Since the reduction in the analytical run time by limited chromatography would lead to inferior analytical quality, we finally decided to employ the method initially developed with CsD as the internal standard, extended chromatography and a total run time of 6 min for routine CsA monitoring.

Specifically, we conclude from our observations that $\mathrm{CsD}$ as an internal standard is applicable for CsA quantification by LC-MS/MS only if extended chromatography is used, at least with the instruments used in the present study. In general, we conclude in accordance with similar observations for the quantification of mycophenolic acid by LC-MS/MS (9) that in analytes that undergo metabolism, non-specificity of LC-MS/MS may arise from metabolites with identical parent and product ions as are found for the target analytes. Therefore, careful verification of the compound specificity of individual MRM transitions using extended chromatography and post-dose patient samples is mandatory for the validation of biomedical high-throughput LC-MS/MS methods that typically employ minimal chromatographic separation.

\section{References}

1. Vogeser M, Fleischer C, Meiser B, Groetzner J, Spöhrer $U$, Seidel D. Quantification of sirolimus by liquid chromatography-tandem mass spectrometry using on-line solid-phase extraction. Clin Chem Lab Med 2002;40:40-5.

2. Volosov A, Napoli KL, Soldin SJ. Simultaneous simple and fast quantification of three major immunosuppressants by liquid chromatography-tandem mass-spectrometry. Clin Biochem 2001;34:285-90.

3. Keevil BG, Tierney DP, Cooper DP, Morris MR. Rapid liquid chromatography-tandem mass spectrometry method for routine analysis of cyclosporin $A$ over an extended concentration range. Clin Chem 2002;48:69-76.

4. Ceglarek U, Lembcke J, Fiedler GM, Werner M, Witzigmann H, Hauss JP, et al. Rapid simultaneous quantification of immunosuppressants in transplant patients by turbulent flow chromatography combined with tandem mass spectrometry. Clin Chim Acta 2004;346:181-90.

5. Koal T, Deters M, Casetta B, Kaever V. Simultaneous determination of four immunosuppressants by means of high speed and robust on-line solid phase extraction-high performance liquid chromatography-tandem mass spectrometry. J Chromatogr B Anal Technol Biomed Life Sci 2004;805:215-22.

6. Tuominen J, Suortti T, Ishikawa K, Lundell J, Koga Y. Separation of cyclosporins by high-performance liquid chromatography and mass spectrometric study of cyclosporin metabolites. Rapid Commun Mass Spectrom 1998; 12:1085-91.

7. Streit F, Armstrong VW, Oellerich M. Mass interferences in quantification of cyclosporine using tandem mass spectrometry without chromatography [abstract]. Ther Drug Monit 2003;25:506

8. Streit F, Armstrong VW, Oellerich M. Rapid liquid chromatography-tandem mass spectrometry routine method for simultaneous determination of sirolimus, everolimus, tacrolimus, and cyclosporin A in whole blood. Clin Chem 2002;48:955-8.

9. Vogeser M, Zachoval R, Spöhrer U, Jacob K. Potential lack of specificity using electrospray tandem-mass spectrometry for the analysis of mycophenolic acid in serum. Ther Drug Monit 2001;23:722-4.

Received October 8, 2004, accepted February 3, 2005 